\title{
Optimal design of multiple dependent state repetitive group sampling plan for Inverse Power Lomax distribution
}

\author{
Rabia Ashraf*1 (D), Nadia Saeed ${ }^{1}$ (D), Kanwal Saleem ${ }^{1}$ (D), Muhammad Aslam² (1D \\ ${ }^{1}$ College of Statistical and Actuarial Sciences, University of the Punjab Lahore-54000, Pakistan \\ ${ }^{2}$ Department of Statistics, Faculty of Science, King Abdulaziz University, Jeddah 21551, Saudi Arabia
}

\begin{abstract}
A sampling plan named as Multiple Dependent State Repetitive Group Sampling (MDSRGS) plan is introduced for a time-truncated life test given that the underlying distribution of the product's lifetime is Inverse Power Lomax distribution (IPLD). The proposed sampling plan is developed with the help of two already developed sampling plans (MDS and RGS). The two-point approach OC function known as the producer's risk and customer's risk is used to determine the parameters of the proposed plan. An optimization method is used for different values of customer's risk and producer's risk, for static values of experiment termination ratio and mean ratio, to find out plan parameters (minimum size of the sample, the number of acceptance and rejection, and the number of successive lots). Tables are created for different known values of shape parameters of Inverse Power Lomax distribution. The efficiency of the proposed MDSRGS plan is examined by conducting a comparative study. To accompany the results, graphs are also used for visualizing the average sample and acceptance probabilities with the specific mean ratio. Two real-life applications are also incorporated to demonstrate the operating procedure of the proposed plan.
\end{abstract}

Mathematics Subject Classification (2020). 62D05, 62P30

Keywords. multiple dependent state sampling, repetitive sampling, OC curve, termination time

\section{Introduction}

In everyday life, consumers consider quality standards an important factor while buying or choosing an item. The factor of maintaining quality standards is not only considered important for consumers but also for an organization, producer, industry, and retail store. It is said that customer's needs and requirements lead to the product quality in a production process.

A product is usually said to be reputed if it leads to quality standards. Maintaining quality standards for the products is a big challenge for manufacturers and producers. In

\footnotetext{
*Corresponding Author.

Email addresses: rabia.ashraf63@yahoo.com (R. Ashraf), nadia.stat@pu.edu.pk (N. Saeed), kanwalsaleem16@gmail.com (K. Saleem), aaslam_ravian@hotmail.com (M. Aslam)

Received: 07.02.2021; Accepted: 04.12.2021
} 
regards to this, they must carefully inspect the product quality before launching it in the market. Certain statistical procedures such as total quality control to ensure the quality of a product is available. The most highlighted factor of a product is its long life which fluctuates the consumer's behavior positively towards buying any product. Therefore, production managers and manufacturers have to put their complete attention and care into every stage of the manufacturing process. In such situations, acceptance sampling designs are more helpful. To explore the quality of submitted lots, acceptance sampling plans are widely used mostly in the production process. The acceptance sampling plan deals with selecting a small sample size from the submitted lot while the inspection procedure is in process and then further tests are applied to it which leads us to decide whether to accept or reject the entire lot according to the results generated from randomly selected items. Acceptance sampling has a disadvantage as it can select a bad lot and can reject a good one due to the selected sample which only describes the small portion of the specified lot. Producer's risk $(\alpha)$ and consumer's risk $(\beta)$ are known as the probabilities of rejecting a good and accepting a bad lot. Acceptable quality level (AQL) is defined as the consistency of product quality level with producer's risk therefore it is understood that the producer will enhance the quality of a product so the producer's risk can be reduced. On the other hand, if the quality level is consistent with the consumer's risk called limiting quality level (LQL) then the consumer will require low chances of defective items so that the consumer risk can be minimized. Confidence level about consumer's risk must have to be incorporated when constructing a sampling plan under a truncated life test. While shaping the plan's parameters, the consumer's risk and producer's risks are permanently associated with the acceptance sampling plan. Moreover, these two risks are called the two-point approach.

Since the product's long lifetime is a charming feature that attracts consumers towards buying that product. The lifetime testing is explored by time truncated life test under some specific conditions. This testing can be conducted for pre-assigned time.

With this method, the chances of accepting faulty products are minimized. A testing lifetime of a product has a time restriction. Testing every product in a lot is timeconsuming so it is advisable to stop the experiment at the pre-assigned time for observing the faulty items. The specific lot will be accepted if the number of failure items coincides with the number of accepted items given that the pre-allotted time has been passed. Similarly, if the number of failure items is more than the defined number of defectives, the lot will not be accepted. Huge literature is available on time truncated life testing for different underlying distributions. A repetitive sampling plan suggested by [3] worked on Weibull distribution given that the shape parameters of the underlying distribution were not unknown. Another acceptance sampling plan was developed by [1] when the product's lifetime followed the transmuted inverse Rayleigh distribution. For Rayleigh distribution, a group chain sampling plan was introduced by [15]. Balamurali et al. [9] came up with a new acceptance sampling plan for life testing which was referred to as the MDS sampling plan whose theoretical distribution was Weibull distribution. Detailed work on this topic is available in $[4,6,12,13]$.

The attribute, variable, continuous and a specific purpose sampling plans are four main categories into which acceptance sampling plan is categorized. More precisely, the main category of acceptance sampling plan is found to be a specific purpose sampling plan [11]. In the situation where lots are submitted serially, the specific purpose sampling plans proved to be helpful and this plan (Specific Purpose sampling plan) was introduced by [17] into which repetitive group sampling (RGS) technique was used to develop the plan. Wortham and Baker [21] proposed a new sampling plan named multiple dependent state (MDS). An extensive literature can be found regarding MDS and RGS plans in [5,7,20,22], etc. Aslam et al. [2] researched to develop the facts which assured the product percentile life under Burr XII distribution by using the MDSRGS plan. 
Acceptance sampling plan of variable type multiple dependent state repetitive (MDSR) was proposed by [18] which was based on two sampling plans such as multiple dependent states (MDS) and Repetitive Group sampling (RGS) plans. Furthermore, process capability was also explored with the help of the MDSRGS plan. Moreover, Balamurali et al. [10] investigated the product lifetime which follows Weibull and gamma distributions as well by using the MDSRGS plan.

Singh [8] proposed a sampling plan used for the scenarios where the product lifetime follows generalized Pareto distribution. In this research, for the sample size, comparisons were made between the single acceptance and the proposed plans. It was studied that the proposed plan requires comparatively less sample size and hence cost-efficient. A multiple deferred state sampling plan was proposed by [16] which was based on the median life of the product given that the lifetime of product belongs to the generalized inverted exponential distribution. Optimal parameters were estimated using an operating characteristic curve. The performance of the proposed plan was found to be better than the existing ones. Another sampling plan named repetitive group sampling plan was proposed by [19] according to which the quality parameter was accessed by median life of a product where the product lifetime follows a generalized inverted exponential distribution. Through a comparative study, the performance of the proposed plan was also accessed which was found to be better than the existing ones. For the lifetime of the product when it follows the inverse Weibull distribution, a multiple deferred state repetitive sampling plan was proposed by [8]. To represent the quality of the product, the true and specified median life ratios were used. Comparative analysis was carried out and significantly better results than the existing plans were observed by the research findings.

The present research paper outlined the truncated life test by using the proposed MDSRGS plan when the underlying distribution of the product's life follows Inverse Power Lomax distribution (IPLD). To establish the facts for assuring the mean life of a product with minimum cost and time were the main objectives of this research. The proposed plan gives the minimum sample size and average sample number (ASN) when compared with existing RGS and SS plans under IPLD. The operating procedure of the proposed plan is described by using two real-life data sets.

In this article, the inverse power lomax distribution is being used. As it is a well-known lifetime distribution used in reliability and life testing problems. As far as our knowledge is concerned, the said distribution is not already being addressed under the MDSRGS plan which justifies its strength.

The study is designed as follows: Section 2 contains the introduction of the underlying distribution. The MDSRGS plan, its operating procedure, and performance measures are defined in Section 3, furthermore product failure probability and sensitivity analysis of MDSRGS plan is also carried out and discussed. Comparison of the proposed plan with already existing plans to determine its efficiency is discussed in Section 4. The analysis and interpretations are discussed in Section 5 while Section 6 comprises of comparative analysis of proposed plan. Section 7 constitutes a practical application. Lastly, the concluding remarks and future recommendations are given.

\section{Inverse Power Lomax distribution}

Hassan and Abd-Allah [14] proposed Inverse Power Lomax distribution (IPLD), in which a transformation $x=\frac{1}{y}$ was used. The authors presented all fundamental properties of the distribution and proved that it can produce better results than many other distributions. Due to this reason, the distribution is used for developing the MDSRGS plan. This distribution has also been derived from the Power Lomax distribution. Following is the pdf and CDF of the specified distribution. 
The pdf of IPL distribution is

$$
g(x ; \delta, \lambda, \eta)=\frac{\delta \eta}{\lambda} x^{-(\eta+1)}\left(1+\frac{x^{-\eta}}{\lambda}\right)^{-\delta-1}, x, \delta, \eta, \lambda>0 .
$$

The CDF of IPL distribution is

$$
G(x ; \delta, \lambda, \eta)=\left(1+\frac{x^{-\eta}}{\lambda}\right)^{-\eta},
$$

where the scale parameter is denoted by $\lambda$ and the shape parameters are denoted by $\delta$ and $\eta$. As it was assumed that shape parameters are not unknown and therefore CDF depends only on $\frac{x}{\lambda}$. The average life of a product given that it follows the Inverse Power Lomax distribution is as follows:

$$
\mu=\frac{\lambda^{\frac{-1}{\eta}}}{\Gamma(\delta)} \Gamma\left(1-\frac{1}{\eta}\right) \Gamma\left(\delta+\frac{1}{\eta}\right) .
$$

Before the termination of the experiment, the failure item's probability under Inverse Power Lomax distribution is given by

$$
P=F\left(t_{0}\right)=\left(1+\frac{t_{0}^{-\eta}}{\lambda}\right)^{-\delta} .
$$

The experiment time $t_{0}=a \mu_{0}$ is explained by average life $\mu_{0}$ where $a$ is constant and also the experiment termination ratio. Therefore, the product failure's probability before the termination time is

$$
P=\left[1+\left(\frac{\delta \Gamma\left(1-\frac{1}{\eta}\right) \Gamma\left(\delta+\frac{1}{\eta}\right)^{-\eta}}{r \Gamma \delta}\right)\right]^{-\delta},
$$

where $P_{1}$ and $P_{2}$ are the probabilities of failure of producer's risk and consumer's risk respectively.

\section{Multiple dependent state repetitive group sampling plan}

\subsection{Operating procedure}

The four parameters $n, c_{1}, c_{2}$ and $m$ are used for the proposed MDSRGS plan. The proposed plan's operating procedure based on a time truncated life test follows the three steps listed below:

Step 1. From the current lot, life testing is applied to a selected sample of size $n$ at a specific time $t_{0}$.

Step 2. Failure items are counted at the experiment termination time $t_{0}$ and these items are then denoted by $d$.

Step 3.The results which are obtained from the life testing procedure decide the acceptance and rejection of the submitted lot as per the following points:

- If $d \leq c_{1}$, the current submitted lot will be accepted.

- If $d>c_{2}$, the submitted lot will be rejected and the life test will be truncated immediately.

- If $c_{1}<d \leq c_{2}$, the current lot will be accepted if in the case of the MDS sampling plan the successive $m$ lots accept at $d \leq c_{1}$. If this condition does not hold, repeat the procedure until the decision is taken on the current lot.

The $n, c_{1}, c_{2}$ and, $m$ are four plan parameters used for the proposed MDSRGS plan. Here $n$ is the sample size, $c_{1}$ is the maximum allowable number of failure items for unconditional acceptance, $c_{2}$ is the maximum additional number of failure items for conditional acceptance and $m$ is the number of successive lots required for making a decision. 
For the specific situations, the proposed MDSRGS plan can be transformed to Repetitive Group Sampling (RGS) plan at $m \rightarrow \infty$, Single Sampling (SS) plan at $c_{2}=c_{1}=c$, and at $m=0$ with $c_{2}$ numbers of acceptance.

\subsection{Performance measurements}

To examine the performance of a sampling plan, different performance measures are used such as average sample number (ASN) and operating characteristic (OC) functions. In this study, we have derived the OC and ASN functions of the MDSRGS plan following the steps provided by [2] and [10].

$P_{1}(p)$ is the probability of accepting the lot that is based on Multiple Dependent Sampling as

$$
P_{1}(p)=P\left(d \leq c_{1}\right)+P\left(c_{1}<d \leq c_{2}\right)\left(P\left(d \leq c_{1}\right)\right)^{m} .
$$

$P_{2}(p)$ is the probability of rejecting the lot that is based on a single sampling as follows:

$$
P_{2}(p)=P\left(d>c_{2}\right)=1-\left(P\left(d \leq c_{2}\right)\right) .
$$

$P_{3}(p)$ is the probability that will be repeated by the sampling as follows:

$$
P_{3}(p)=P\left(c_{1}<k d \leq c_{2}\right)\left(1-\left(P\left(d \leq c_{1}\right)\right)^{m}\right) .
$$

$P\left(d \leq c_{1}\right)$ and $P\left(c_{1}<d \leq c_{2}\right)$ are described as follows under the binomial distribution.

$$
P\left(d \leq c_{1}\right)=\sum_{d=0}^{c_{1}} n_{C_{d}} p^{d}(1-p)^{n-d}
$$

and

$$
P\left(c_{1}<d \leq c_{2}\right)=\sum_{d=c_{1}+1}^{c_{2}} n_{C_{d}} p^{d}(1-p)^{n-d} .
$$

Therefore, the operating characteristic and ASN functions of the MDSRGS plan are given below which is based on the time truncated life test under Inverse Power Lomax Distribution, following [2] and [10].

$$
P_{a}(p)=\frac{P\left(d \leq c_{1}\right)+P\left(c_{1}<d \leq c_{2}\right)\left(P\left(d \leq c_{1}\right)\right)^{m}}{1-\left(P\left(c_{1}<d \leq c_{2}\right)\right)\left(1-\left(P\left(d \leq c_{1}\right)\right)^{m}\right)}
$$

and

$$
A S N(p)=\frac{n}{1-\left(P\left(c_{1}<d \leq c_{2}\right)\right)\left(1-\left(P\left(d \leq c_{1}\right)\right)^{m}\right)} .
$$

In this study, the shape parameters of Inverse Power Lomax Distribution are assumed to be known as provided by Hassan and Abd-Allah [14] but practically, these values are not available. In this situation, the production process history can be useful to estimate the shape parameters values. Generally, the producers maintain the history of production process.

\subsection{Design methodology}

Although for inspecting the lot, different plans have been designed but a plan with a small size of the sample is favored for the disposition of the lot. Minimum sample-sized plans decrease the inspection cost so this type of plan is known as economical sampling plan. The design of the MDSRGS plan also depends on the minimum size of the sample. So the significant purpose of this study is to minimize the average of ASN at Acceptable Quality Level (AQL) and Limiting Quality Level (LQL). The design parameters of the MDSRGS plan are estimated by using the optimization problem given as

$$
\begin{gathered}
\text { minimize } \frac{1}{2}\left[A S N\left(p_{1}\right)+A S N\left(p_{2}\right)\right], \\
\text { subject to } P_{a}\left(p_{1}\right) \geq 1-\alpha,
\end{gathered}
$$




$$
P_{a}\left(p_{1}\right) \leq \beta, n>1, m \geq 1, c_{2}>c_{1} \geq 0
$$

The average sample number of MDSRGS plan based on the time truncated life for Inverse Power Lomax Distribution can be obtained by using the above equation.

$$
A S N\left(p_{1}\right)=\frac{n}{1-\sum_{d=c_{1}+1}^{c_{2}} n_{C_{d}} p_{1}^{d}\left(1-p_{1}\right)^{n-d}\left(1-\left(\sum_{d=0}^{c_{1}} n_{C_{d}} p_{1}^{d}\left(1-p_{1}\right)^{n-d}\right)^{m}\right)}
$$

and

$$
A S N\left(p_{2}\right)=\frac{n}{1-\sum_{d=c_{1}+1}^{c_{2}} n_{C_{d}} p_{2}^{d}\left(1-p_{2}\right)^{n-d}\left(1-\left(\sum_{d=0}^{c_{1}} n_{C_{d}} p_{2}^{d}\left(1-p_{2}\right)^{n-d}\right)^{m}\right)} .
$$

The acceptance probabilities of the MDSRGS plan based on time truncated life test under Inverse Power Lomax Distribution can be obtained by using above Equation (3.9).

$$
\begin{aligned}
& P_{a}\left(p_{1}\right)=\frac{\sum_{d=0}^{c_{1}} n_{C_{d}} p_{1}^{d}\left(1-p_{1}\right)^{n-d}+\sum_{d=c_{1}+1}^{c_{2}} n_{C_{d}} p_{1}\left(1-p_{1}\right)^{n-d}\left(\sum_{d=0}^{c_{1}} n_{C_{d}} p_{2}^{d}\left(1-p_{2}\right)^{n-d}\right)^{m}}{1-\sum_{d=c_{1}+1}^{c_{2}} n_{C_{d}} p_{2}^{d}\left(1-p_{2}\right)^{n-d}\left(1-\left(\sum_{d=0}^{c_{1}} n_{C_{d}} p_{2}^{d}\left(1-p_{2}\right)^{n-d}\right)^{m}\right)} \\
& P_{a}\left(p_{2}\right)=\frac{\sum_{d=c_{1}+1}^{c_{1}} n_{C_{d}} p_{2}^{d}\left(1-p_{2}\right)^{n-d}+\sum_{d=c_{1}+1}^{c_{2}} n_{C_{d}} p_{2}\left(1-p_{2}\right)^{n-d}\left(\sum_{d=0}^{c_{1}} n_{C_{d}} p_{2}^{d}\left(1-p_{2}\right)^{n-d}\right)^{m}}{1-\sum_{d=c_{1}+1}^{c_{2}} n_{C_{d}} p_{2}^{d}\left(1-p_{2}\right)^{n-d}\left(1-\left(\sum_{d=0}^{c_{1}} n_{C_{d}} p_{2}^{d}\left(1-p_{2}\right)^{n-d}\right)^{m}\right)}
\end{aligned}
$$

Here, the mean ratio represents the product's quality which is computed by dividing the true mean life by the specified mean life. Additionally, $p_{1}$ is the probability of failure that corresponds to the mean ratios $\frac{\mu}{\mu_{0}}=2,4,6,8,10$ is known as Acceptance Quality Level $(\mathrm{AQL})$ while $p_{2}$ is the probability of failure that corresponds to the mean ratio $\frac{\mu}{\mu_{0}}=1$ is named as Limiting Quality Level (LQL).

\section{Simulation study}

Following steps are followed to construct a code of MDSRGS Plan under IPL distribution to attain the design parameters:

Step 1 First of all the values of the shape parameters $\delta$ and $\eta$, producer's risk $(\alpha)$, consumer's risk $(\beta)$ and termination ratio $(a)$ are stated under Inverse Power Lomax Distribution. The maximum values of ASN are fixed and that is known as $A S N_{m}$.

Step 2 Values of plan parameters are fixed as $n=2, c_{1}=0, c_{2}=c_{1}+1, m=1$.

Step 3 The failure probabilities for Inverse Power Lomax distribution corresponding to the specified mean ratios $\frac{\mu}{\mu_{0}}=2,4,6,8,10$ are obtained by using Equation (2.5) and is denoted as AQL $\left(p_{1}\right)$. In the same way, the failure probability for Inverse Power Lomax distribution with the mean ratio $\frac{\mu}{\mu_{0}}=1$ is found by using Equation (2.5) and it is denoted as LQL $\left(p_{2}\right)$.

Step 4 The probability of acceptance at AQL can be obtained by using Equation (3.10), it is denoted as $P_{a}\left(p_{1}\right)$ and the ASN is calculated at AQL by using Equation (3.8) which is denoted as $A S N\left(p_{1}\right)$. Similarly, the probability of acceptance at LQL can be obtained by using Equation 3.11, it is denoted as $P_{a}\left(p_{2}\right)$ and the ASN is calculated at LQL by using Equation (3.9) and it is denoted as $A S N\left(p_{2}\right)$.

Step 5 Required optimal parameters are obtained when the conditions $P_{a}\left(p_{1}\right) \geq 1-\alpha$ and $P_{a}\left(p_{2}\right) \leq \beta$ are fulfilled.

Step 6 In this step value of $\frac{1}{2}\left(A S N\left(p_{1}\right)+A S N\left(p_{2}\right)\right)$ is compared with $A S N_{m}$. If the condition $\frac{1}{2}\left(A S N\left(p_{1}\right)+A S N\left(p_{2}\right)\right) \leq A S N_{m}$ is satisfied then the required $A S N_{m}$ is obtained. Until the required minimum value of $A S N_{m}$ is obtained, repeat the procedure for different arrangements of $n, c_{1}, c_{2}, m$.

Step 7 The required optimal parameters such as $n, c_{1}, c_{2}$ and $\mathrm{m}$ are obtained when such $A S N_{m}$ is existed. If not then steps $4-6$ are repeated until the required optimal parameters such as $n, c_{1}, c_{2}$ and, $m$ are obtained. 
To obtain the design parameters of the proposed plan under IPL distribution, the $\mathrm{R}$ code is generated. The range of parameters used is $n=2(1) 100, c_{1}=0(1) 20, c_{2}=$ $c_{1}+1(1) c_{1}+10, m=1(1) 6$. The consumer and producer risks $(\beta=0.25,0.10,0.05,0.01$ and $\alpha=0.05)$ with the experiment termination ratios $(a=0.5$ and $a=1.0)$, are satisfied given that design parameters are calculated. The proposed plan's designed parameters following Inverse Power Lomax distribution with a different combination of shape parameters $\delta=$ $0.7, \eta=3, \delta=1.5, \eta=3, \delta=2, \eta=1.8, \delta=2.5, \eta=2$ are tabulated in the proceeding section. Lot acceptance probabilities at AQL and LQL are presented in the next two tables. Moreover, for the Inverse Power Lomax distribution, the sensitivity analysis of the plan was also carried out.

\section{Analysis and interpretation}

To estimate the chances of failure items $p_{1}$ and $p_{2}$ the proposed plan following the Inverse Power Lomax distribution is used. AQL represents the mean ratios as $\frac{\mu}{\mu_{0}}=2,4,6,8,10$ which represent the probability of failure denoted by $p_{1}$ whereas; LQL represents the mean ratio $\frac{\mu}{\mu_{0}}=1$ which is also the probability of failure $p_{2}$. The design parameters are studied in such a way that both consumer's risk $\beta=0.25,0.10,0.05,0.01$ and producer's risk $\alpha=0.05$ are satisfied at the same time. The experiment ratios are defined as $a=0.5$ and $a=1.0$ with combinations of fixed values of two parameters $\delta=0.7, \eta=3, \delta=$ $1.5, \eta=3, \delta=2, \eta=1.8, \delta=2.5, \eta=2$ are observed as shape parameters. Various combinations of $\delta$ and $\eta$ which are the shape parameters are presented in Tables 1-4. The producer's risk is kept fix whereas four levels of consumer's risk were included. The plan parameters $n, c_{1}, c_{2}, m$ and ASN are also incorporated in the tables. It is stated that the sample size will be decreased as the mean and experiment termination ratio increase. It was noticed that the sample size and ASN increased when the termination ratio tends to 0.5. Contradictory of this at 1.0 the sample size and ASN will be decreased. So, based on sensitivity analysis it was concluded that whenever the termination ratio goes from 0.5 to 1.0 the sample size and ASN both are supposed to decrease. By increasing sample size and ASN, consumer risk can be decreased.

Table 1. MDSRGS Plan parameters under IPL distribution at $\delta=0.7, \eta=3$.

\begin{tabular}{|c|c|c|c|c|c|c|c|c|c|c|c|}
\hline \multirow{3}{*}{$\beta$} & & \multicolumn{9}{|c|}{$\mathbf{a}=\mathbf{0 . 5}$} & \multicolumn{6}{|c|}{$\mathbf{a}=\mathbf{1 . 0}$} \\
\hline & $\frac{\mathbf{n}}{\mu_{0}}$ & $\mathbf{c}_{\mathbf{1}}$ & $\mathbf{c}_{\boldsymbol{2}}$ & $\mathbf{m}$ & $\mathbf{A S N}$ & $\mathbf{n}$ & $\mathbf{c}_{\boldsymbol{1}}$ & $\mathbf{c}_{\boldsymbol{2}}$ & $\mathbf{m}$ & $\mathbf{A S N}$ \\
\hline 0.25 & 2 & 13 & 1 & 2 & 2 & 15.2 & 4 & 1 & 2 & 2 & 5.1 \\
& 4 & 9 & 0 & 1 & 1 & 9.5 & 3 & 0 & 1 & 1 & 3.5 \\
& 6 & 8 & 0 & 1 & 1 & 9.4 & 3 & 0 & 1 & 1 & 3.5 \\
& 8 & 8 & 0 & 1 & 1 & 9.4 & 3 & 0 & 1 & 1 & 3.5 \\
& 10 & 8 & 0 & 1 & 1 & 9.4 & 3 & 0 & 1 & 1 & 3.5 \\
\hline 0.10 & 2 & 13 & 0 & 2 & 1 & 19 & 6 & 1 & 3 & 1 & 8.2 \\
& 4 & 11 & 0 & 1 & 1 & 12.3 & 3 & 0 & 1 & 1 & 3.5 \\
& 6 & 11 & 0 & 1 & 1 & 12.2 & 3 & 0 & 1 & 1 & 3.5 \\
& 8 & 11 & 0 & 1 & 1 & 12.2 & 3 & 0 & 1 & 1 & 3.5 \\
& 10 & 11 & 0 & 1 & 1 & 12.2 & 3 & 0 & 1 & 1 & 3.5 \\
\hline 0.05 & 2 & 22 & 1 & 3 & 1 & 26.3 & 6 & 1 & 3 & 2 & 8.7 \\
& 4 & 13 & 0 & 1 & 1 & 14.2 & 4 & 0 & 1 & 1 & 4.4 \\
& 6 & 13 & 0 & 1 & 1 & 14 & 4 & 0 & 1 & 1 & 4.3 \\
& 8 & 13 & 0 & 1 & 1 & 14 & 4 & 0 & 1 & 1 & 4.3 \\
& 10 & 13 & 0 & 1 & 1 & 14 & 4 & 0 & 1 & 1 & 4.3 \\
\hline 0.01 & 2 & 29 & 1 & 4 & 1 & 36.6 & 8 & 1 & 4 & 2 & 13.2 \\
& 4 & 19 & 0 & 1 & 1 & 19.9 & 5 & 0 & 1 & 1 & 5.3 \\
& 6 & 19 & 0 & 1 & 1 & 19.5 & 5 & 0 & 1 & 1 & 5.2 \\
& 8 & 19 & 0 & 1 & 1 & 19.5 & 5 & 0 & 1 & 1 & 5.2 \\
& 10 & 19 & 0 & 1 & 1 & 19.4 & 5 & 0 & 1 & 1 & 5.2 \\
\hline
\end{tabular}


Table 2. MDSRGS Plan parameters under IPL distribution at $\delta=1.5, \eta=3$.

\begin{tabular}{|c|c|c|c|c|c|c|c|c|c|c|c|}
\hline \multirow{3}{*}{$\beta$} & \multirow{2}{*}{$\boldsymbol{\mu}$} & \multicolumn{9}{|c|}{$\mathbf{a}=\mathbf{0 . 5}$} & \multicolumn{5}{|c|}{$\mathbf{a}=\mathbf{1 . 0}$} \\
\hline & $\frac{\mathbf{n}}{\mu_{0}}$ & $\mathbf{c}_{\boldsymbol{1}}$ & $\mathbf{c}_{\mathbf{2}}$ & $\mathbf{m}$ & $\mathbf{A S N}$ & $\mathbf{n}$ & $\mathbf{c}_{\mathbf{1}}$ & $\mathbf{c}_{\boldsymbol{2}}$ & $\mathbf{m}$ & $\mathbf{A S N}$ \\
\hline 0.25 & 2 & 12 & 0 & 1 & 2 & 14.8 & 2 & 0 & 1 & 2 & 2.9 \\
& 4 & 12 & 0 & 1 & 2 & 14.7 & 2 & 0 & 1 & 2 & 2.8 \\
& 6 & 12 & 0 & 1 & 2 & 14.7 & 2 & 0 & 1 & 2 & 2.8 \\
& 8 & 12 & 0 & 1 & 2 & 14.7 & 2 & 0 & 1 & 2 & 2.8 \\
& 10 & 12 & 0 & 1 & 2 & 14.7 & 2 & 0 & 1 & 2 & 2.8 \\
\hline 0.10 & 2 & 17 & 0 & 1 & 2 & 19.6 & 5 & 1 & 2 & 1 & 5.6 \\
& 4 & 17 & 0 & 1 & 2 & 19.3 & 3 & 0 & 1 & 1 & 3.5 \\
& 6 & 17 & 0 & 1 & 2 & 19.3 & 3 & 0 & 1 & 1 & 3.5 \\
& 8 & 17 & 0 & 1 & 2 & 19.3 & 3 & 0 & 1 & 1 & 3.5 \\
& 10 & 17 & 0 & 1 & 2 & 19.3 & 3 & 0 & 1 & 1 & 3.5 \\
\hline 0.05 & 2 & 21 & 0 & 1 & 2 & 23.3 & 4 & 0 & 2 & 1 & 6 \\
& 4 & 21 & 0 & 1 & 2 & 22.7 & 4 & 0 & 1 & 1 & 4.3 \\
& 6 & 21 & 0 & 1 & 2 & 22.7 & 4 & 0 & 1 & 1 & 4.3 \\
& 8 & 21 & 0 & 1 & 2 & 22.7 & 4 & 0 & 1 & 1 & 4.3 \\
& 10 & 21 & 0 & 1 & 2 & 22.7 & 4 & 0 & 1 & 1 & 4.3 \\
\hline 0.01 & 2 & 31 & 0 & 1 & 1 & 32.6 & 5 & 0 & 2 & 1 & 6.7 \\
& 4 & 31 & 0 & 1 & 1 & 31.7 & 5 & 0 & 1 & 1 & 5.1 \\
& 6 & 31 & 0 & 1 & 1 & 31.7 & 5 & 0 & 1 & 1 & 5.1 \\
& 8 & 31 & 0 & 1 & 1 & 31.7 & 5 & 0 & 1 & 1 & 5.1 \\
& 10 & 31 & 0 & 1 & 1 & 31.7 & 5 & 0 & 1 & 17 & 5.1 \\
\hline
\end{tabular}

Table 3. MDSRGS Plan parameters under IPL distribution at $\delta=2, \eta=1.8$.

\begin{tabular}{|c|c|c|c|c|c|c|c|c|c|c|c|}
\hline \multirow{3}{*}{$\beta$} & & \multicolumn{7}{|c|}{$\mathbf{a}=\mathbf{0 . 5}$} & \multicolumn{5}{|c|}{$\mathbf{a}=\mathbf{1 . 0}$} \\
\cline { 2 - 10 }$\mu_{0}$ & $\mathbf{n}$ & $\mathbf{c}_{\mathbf{1}}$ & $\mathbf{c}_{\boldsymbol{2}}$ & $\mathbf{m}$ & $\mathbf{A S N}$ & $\mathbf{n}$ & $\mathbf{c}_{\boldsymbol{1}}$ & $\mathbf{c}_{\boldsymbol{2}}$ & $\mathbf{m}$ & $\mathbf{A S N}$ \\
\hline 0.25 & 2 & 7 & 1 & 2 & 1 & 8.1 & 4 & 1 & 3 & 1 & 7.9 \\
& 4 & 4 & 0 & 1 & 1 & 4.8 & 2 & 0 & 1 & 1 & 2.6 \\
& 6 & 4 & 0 & 1 & 1 & 4.8 & 2 & 0 & 1 & 1 & 2.6 \\
& 8 & 4 & 0 & 1 & 1 & 4.8 & 2 & 0 & 1 & 1 & 2.6 \\
& 10 & 4 & 0 & 1 & 1 & 4.8 & 2 & 0 & 1 & 1 & 2.6 \\
\hline 0.10 & 2 & 6 & 0 & 2 & 2 & 10.6 & 9 & 4 & 6 & 2 & 12.3 \\
& 4 & 5 & 0 & 1 & 2 & 5.8 & 3 & 0 & 1 & 1 & 3.4 \\
& 6 & 5 & 0 & 1 & 2 & 5.8 & 3 & 0 & 1 & 1 & 3.3 \\
& 8 & 5 & 0 & 1 & 2 & 5.8 & 3 & 0 & 1 & 1 & 3.3 \\
& 10 & 5 & 0 & 1 & 2 & 5.8 & 3 & 0 & 1 & 1 & 3.3 \\
\hline 0.05 & 2 & 11 & 1 & 3 & 1 & 13.5 & 9 & 3 & 6 & 1 & 13.8 \\
& 4 & 7 & 0 & 1 & 1 & 7.5 & 3 & 0 & 1 & 1 & 3.4 \\
& 6 & 7 & 0 & 1 & 1 & 7.5 & 3 & 0 & 1 & 1 & 3.3 \\
& 8 & 7 & 0 & 1 & 1 & 7.5 & 3 & 0 & 1 & 1 & 3.3 \\
& 10 & 7 & 0 & 1 & 1 & 7.5 & 3 & 0 & 1 & 1 & 3.3 \\
\hline 0.01 & 2 & 15 & 1 & 4 & 1 & 19.5 & 15 & 6 & 9 & 1 & 17.9 \\
& 4 & 9 & 0 & 1 & 1 & 9.3 & 4 & 0 & 2 & 1 & 5.1 \\
& 6 & 9 & 0 & 1 & 1 & 9.3 & 4 & 0 & 1 & 1 & 4.1 \\
& 8 & 9 & 0 & 1 & 1 & 9.3 & 4 & 0 & 1 & 1 & 4.1 \\
& 10 & 9 & 0 & 1 & 1 & 9.3 & 4 & 0 & 1 & 1 & 4.1 \\
\hline
\end{tabular}

Acceptance probabilities related to the submitted lot for various categories of shape parameters $\delta=0.7, \eta=3, \delta=1.5, \eta=3, \delta=2, \eta=1.8, \delta=2.5, \eta=2$ are organized in Tables 5 and 6 . In each table under the specified value of producers' risk $\alpha=0.05$ and the four distinct values of consumer's risk $\beta=0.25,0.10,0.05,0.01$ are taken. Two points $a=0.5$ and $a=1.0$ were also kept fixed for the experiment termination ratio. In addition to this, various mean ratios $\frac{\mu}{\mu_{0}}=2,4,6,8,10$ were considered to obtain the probabilities. Lastly, it was found that acceptance probability $P_{a}\left(p_{1}\right)$ at AQL increased as 
the mean ratio increased for all the categories of shape parameters and termination ratios. Hence it is clear that if the producer of the process raises the quality to a satisfactory level then the producer's risk will be decreased while acceptance probability $P_{a}\left(p_{2}\right)$ at LQL and consumer's risk in most of the cases increased as the mean ratio increases.

Table 4. MDSRGS Plan parameters under IPL distribution at $\delta=2.5, \eta=2$.

\begin{tabular}{|c|c|c|c|c|c|c|c|c|c|c|c|}
\hline \multirow{3}{*}{$\beta$} & & \multicolumn{7}{|c|}{$\mathbf{a}=\mathbf{0 . 5}$} & \multicolumn{5}{|c|}{$\mathbf{a}=\mathbf{1 . 0}$} \\
\cline { 3 - 10 }$\mu_{0}$ & $\mathbf{n}$ & $\mathbf{c}_{\mathbf{1}}$ & $\mathbf{c}_{\boldsymbol{2}}$ & $\mathbf{m}$ & $\mathbf{A S N}$ & $\mathbf{n}$ & $\mathbf{c}_{\mathbf{1}}$ & $\mathbf{c}_{\boldsymbol{2}}$ & $\mathbf{m}$ & $\mathbf{A S N}$ \\
\hline 0.25 & 2 & 5 & 0 & 1 & 2 & 6.5 & 5 & 2 & 3 & 1 & 5.9 \\
& 4 & 5 & 0 & 1 & 2 & 6.2 & 2 & 0 & 1 & 1 & 2.6 \\
& 6 & 5 & 0 & 1 & 2 & 6.2 & 2 & 0 & 1 & 1 & 2.6 \\
& 8 & 5 & 0 & 1 & 2 & 6.2 & 2 & 0 & 1 & 1 & 2.6 \\
& 10 & 5 & 0 & 1 & 2 & 6.2 & 2 & 0 & 1 & 1 & 2.6 \\
\hline 0.10 & 2 & 8 & 0 & 2 & 2 & 12.1 & 7 & 2 & 4 & 1 & 9 \\
& 4 & 7 & 0 & 1 & 1 & 7.9 & 3 & 0 & 1 & 1 & 3.3 \\
& 6 & 7 & 0 & 1 & 1 & 7.9 & 3 & 0 & 1 & 1 & 3.3 \\
& 8 & 7 & 0 & 1 & 1 & 7.9 & 3 & 0 & 1 & 1 & 3.3 \\
& 10 & 7 & 0 & 1 & 1 & 7.9 & 3 & 0 & 1 & 1 & 3.3 \\
\hline 0.05 & 2 & 9 & 0 & 2 & 2 & 12.9 & 7 & 2 & 4 & 1 & 9 \\
& 4 & 8 & 0 & 1 & 3 & 8.8 & 3 & 0 & 1 & 1 & 3.3 \\
& 6 & 8 & 0 & 1 & 3 & 8.8 & 3 & 0 & 1 & 1 & 3.3 \\
& 8 & 8 & 0 & 1 & 3 & 8.8 & 3 & 0 & 1 & 1 & 3.3 \\
& 10 & 8 & 0 & 1 & 3 & 8.8 & 3 & 0 & 1 & 1 & 3.3 \\
\hline 0.01 & 2 & 13 & 0 & 2 & 1 & 16.1 & 11 & 3 & 6 & 1 & 13.9 \\
& 4 & 12 & 0 & 1 & 1 & 12.3 & 4 & 0 & 1 & 1 & 4.2 \\
& 6 & 12 & 0 & 1 & 1 & 12.3 & 4 & 0 & 1 & 1 & 4.1 \\
& 8 & 12 & 0 & 1 & 1 & 12.3 & 4 & 0 & 1 & 1 & 4.1 \\
& 10 & 12 & 0 & 1 & 1 & 12.3 & 4 & 0 & 1 & 1 & 4.1 \\
\hline
\end{tabular}

Table 5. OC values of the proposed plan for IPL distribution at $\delta=0.7, \eta=3$ and $\delta=1.5, \eta=3$.

\begin{tabular}{|c|c|c|c|c|c|c|c|c|c|}
\hline \multirow[b]{3}{*}{$\beta$} & \multirow[b]{3}{*}{$\frac{\mu}{\mu_{0}}$} & \multicolumn{4}{|c|}{$\delta=0.7, \eta=3$} & \multicolumn{4}{|c|}{$\delta=1.5, \eta=3$} \\
\hline & & \multicolumn{2}{|c|}{$a=0.5$} & \multicolumn{2}{|c|}{$a=1.0$} & \multicolumn{2}{|c|}{$a=0.5$} & \multicolumn{2}{|c|}{$a=1.0$} \\
\hline & & $P_{a}\left(p_{1}\right)$ & $P_{a}\left(p_{2}\right)$ & $P_{a}\left(p_{1}\right)$ & $P_{a}\left(p_{2}\right)$ & $P_{a}\left(p_{1}\right)$ & $P_{a}\left(p_{2}\right)$ & $P_{a}\left(p_{1}\right)$ & $P_{a}\left(p_{2}\right)$ \\
\hline \multirow[t]{5}{*}{0.25} & 2 & 0.9649 & 0.2370 & 0.9594 & 0.2234 & 0.9945 & 0.2465 & 0.9777 & 0.2393 \\
\hline & 4 & 0.9952 & 0.2312 & 0.9905 & 0.0837 & 1.0000 & 0.2465 & 0.9999 & 0.2393 \\
\hline & 6 & 0.9991 & 0.2312 & 0.9982 & 0.0837 & 1.0000 & 0.2465 & 1.0000 & 0.2393 \\
\hline & 8 & 0.9997 & 0.2312 & 0.9995 & 0.0837 & 1.0000 & 0.2465 & 1.0000 & 0.2393 \\
\hline & 10 & 0.9999 & 0.2312 & 0.9998 & 0.0837 & 1.0000 & 0.2465 & 1.0000 & 0.2393 \\
\hline \multirow[t]{5}{*}{0.10} & 2 & 0.9542 & 0.0794 & 0.9696 & 0.0599 & 0.9888 & 0.0974 & 0.9772 & 0.0799 \\
\hline & 4 & 0.9908 & 0.0890 & 0.9905 & 0.0837 & 1.0000 & 0.0974 & 0.9997 & 0.0711 \\
\hline & 6 & 0.9983 & 0.0890 & 0.9982 & 0.0837 & 1.0000 & 0.0974 & 1.0000 & 0.0711 \\
\hline & 8 & 0.9995 & 0.0890 & 0.9995 & 0.0837 & 1.0000 & 0.0974 & 1.0000 & 0.0711 \\
\hline & 10 & 0.9998 & 0.0890 & 0.9998 & 0.0837 & 1.0000 & 0.0974 & 1.0000 & 0.0711 \\
\hline \multirow[t]{5}{*}{0.05} & 2 & 0.9627 & 0.0417 & 0.9659 & 0.0451 & 0.9828 & 0.0484 & 0.9875 & 0.0382 \\
\hline & 4 & 0.9871 & 0.0481 & 0.9815 & 0.0239 & 1.0000 & 0.0484 & 0.9995 & 0.0195 \\
\hline & 6 & 0.9976 & 0.0481 & 0.9965 & 0.0239 & 1.0000 & 0.0484 & 1.0000 & 0.0195 \\
\hline & 8 & 0.9993 & 0.0481 & 0.999 & 0.0239 & 1.0000 & 0.0484 & 1.0000 & 0.0195 \\
\hline & 10 & 0.9997 & 0.0481 & 0.9996 & 0.0239 & 1.0000 & 0.0484 & 1.0000 & 0.0195 \\
\hline \multirow[t]{5}{*}{0.01} & 2 & 0.9713 & 0.0085 & 0.9688 & 0.0076 & 0.9641 & 0.0099 & 0.9693 & 0.0088 \\
\hline & 4 & 0.9726 & 0.0087 & 0.9698 & 0.0077 & 0.9999 & 0.0099 & 0.9991 & 0.0061 \\
\hline & 6 & 0.9948 & 0.0087 & 0.9943 & 0.0077 & 1.0000 & 0.0099 & 1.0000 & 0.0061 \\
\hline & 8 & 0.9984 & 0.0087 & 0.9983 & 0.0077 & 1.0000 & 0.0099 & 1.0000 & 0.0061 \\
\hline & 10 & 0.9994 & 0.0087 & 0.9993 & 0.0077 & 1.0000 & 0.0099 & 1.0000 & 0.0061 \\
\hline
\end{tabular}


Table 6. OC values of the proposed plan for IPL distribution at $\delta=2, \eta=1.8$ and $\delta=2.5, \eta=2$.

\begin{tabular}{|c|c|c|c|c|c|c|c|c|c|}
\hline \multirow[b]{3}{*}{$\beta$} & \multirow[b]{3}{*}{$\frac{\mu}{\mu_{0}}$} & \multicolumn{4}{|c|}{$\delta=2, \eta=1.8$} & \multicolumn{4}{|c|}{$\delta=2.5, \eta=2$} \\
\hline & & \multicolumn{2}{|c|}{$a=0.5$} & \multicolumn{2}{|c|}{$a=1.0$} & \multicolumn{2}{|c|}{$a=0.5$} & \multicolumn{2}{|c|}{$a=1.0$} \\
\hline & & $P_{a}\left(p_{1}\right)$ & $P_{a}\left(p_{2}\right)$ & $P_{a}\left(p_{1}\right)$ & $P_{a}\left(p_{2}\right)$ & $P_{a}\left(p_{1}\right)$ & $P_{a}\left(p_{2}\right)$ & $P_{a}\left(p_{1}\right)$ & $P_{a}\left(p_{2}\right)$ \\
\hline \multirow[t]{5}{*}{0.25} & 2 & 0.962 & 0.2324 & 0.9607 & 0.2317 & 0.9728 & 0.214 & 0.9561 & 0.2387 \\
\hline & 4 & 0.9984 & 0.2296 & 0.9863 & 0.1437 & 0.9999 & 0.214 & 0.9972 & 0.1755 \\
\hline & 6 & 0.9999 & 0.2296 & 0.9985 & 0.1437 & 1.0000 & 0.214 & 0.9999 & 0.1755 \\
\hline & 8 & 1.0000 & 0.2296 & 0.9997 & 0.1437 & 1.0000 & 0.214 & 1.0000 & 0.1755 \\
\hline & 10 & 1.0000 & 0.2296 & 0.9999 & 0.1437 & 1.0000 & 0.214 & 1.0000 & 0.1755 \\
\hline \multirow[t]{5}{*}{0.10} & 2 & 0.9629 & 0.0825 & 0.9666 & 0.0896 & 0.9917 & 0.0759 & 0.9501 & 0.0383 \\
\hline & 4 & 0.9974 & 0.0964 & 0.9604 & 0.0232 & 0.9998 & 0.0939 & 0.9918 & 0.0309 \\
\hline & 6 & 0.9998 & 0.0964 & 0.9955 & 0.0232 & 1.0000 & 0.0939 & 0.9996 & 0.0309 \\
\hline & 8 & 1.0000 & 0.0964 & 0.9992 & 0.0232 & 1.0000 & 0.0939 & 1.0000 & 0.0309 \\
\hline & 10 & 1.0000 & 0.0964 & 0.9998 & 0.0232 & 1.0000 & 0.0939 & 1.0000 & 0.0309 \\
\hline \multirow[t]{5}{*}{0.05} & 2 & 0.9666 & 0.0426 & 0.9596 & 0.0281 & 0.9875 & 0.0445 & 0.9501 & 0.0383 \\
\hline & 4 & 0.9946 & 0.0315 & 0.9604 & 0.0232 & 0.9997 & 0.0499 & 0.9918 & 0.0309 \\
\hline & 6 & 0.9996 & 0.0315 & 0.9955 & 0.0232 & 1.0000 & 0.0499 & 0.9996 & 0.0309 \\
\hline & 8 & 0.9999 & 0.0315 & 0.9992 & 0.0232 & 1.0000 & 0.0499 & 1.0000 & 0.0309 \\
\hline & 10 & 1.0000 & 0.0315 & 0.9998 & 0.0232 & 1.0000 & 0.0499 & 1.0000 & 0.0309 \\
\hline \multirow[t]{5}{*}{0.01} & 2 & 0.9700 & 0.0061 & 0.9516 & 0.0074 & 0.9633 & 0.0076 & 0.9535 & 0.0037 \\
\hline & 4 & 0.9909 & 0.0096 & 0.9936 & 0.0078 & 0.9994 & 0.0094 & 0.9840 & 0.0070 \\
\hline & 6 & 0.9993 & 0.0096 & 0.9911 & 0.0049 & 1.0000 & 0.0094 & 0.9993 & 0.0070 \\
\hline & 8 & 0.9999 & 0.0096 & 0.9984 & 0.0049 & 1.0000 & 0.0094 & 0.9999 & 0.0070 \\
\hline & 10 & 1.0000 & 0.0096 & 0.9996 & 0.0049 & 1.0000 & 0.0094 & 1.0000 & 0.0070 \\
\hline
\end{tabular}

To have the visual understanding, the graphs are inserted to represent the relationship of ASN versus mean ratio $(r)$ and OC function's acceptance probabilities with mean ratio $(r)$ based on various producer's risk levels and several combinations of shape parameters $\delta$ and $\eta$, such as the product lifetime distribution is IPL associated with two different experiment termination ratios. Figure 1 (panel a-b) exhibits the relationship of ASN and mean ratio $(r)$ under Inverse Power Lomax distribution which is found to be inversely associated as the mean ratio becomes larger, the average sample number (ASN) gets smaller given that the consumer's risk and experiment termination ratios were pre-defined $(\beta=0.25,0.10,0.05,0.01$ and $a=0.5$ and $\alpha=1.0)$.

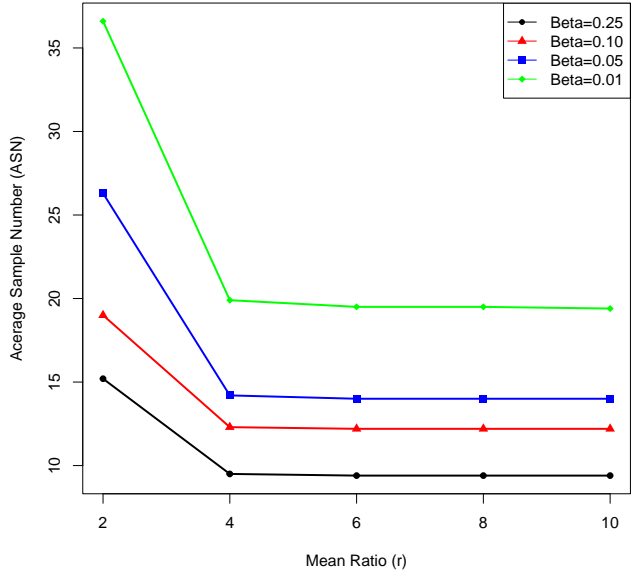

(a) $\delta=0.7, \eta=3$ and $a=0.5$

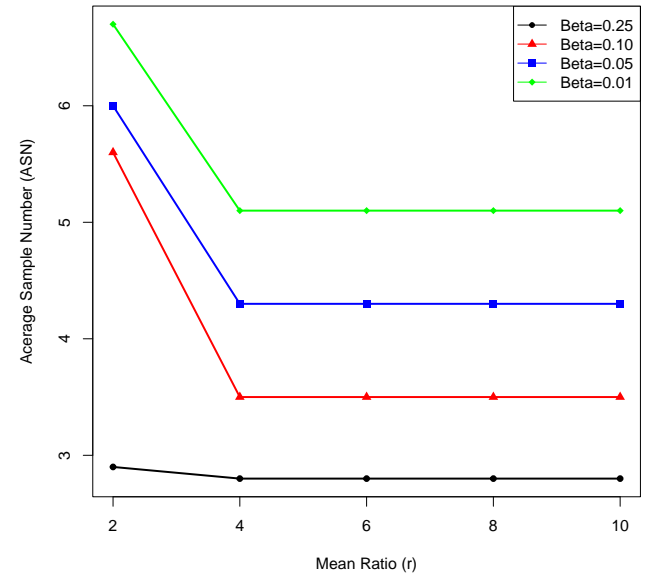

(b) $\delta=1.5, \eta=3$ and $a=1.0$

Figure 1. Average sample number versus mean ratio. 
Figure 2 (panels a-b) are constructed to show the relationship between acceptance probabilities of OC function and mean ratio (r) under Inverse Power Lomax distribution and with different categories of shape parameters. There is a direct or positive relationship seen as the probabilities increases, the mean ratio also tends to increase under the defined customer's risk and experiment termination ratios $(\beta=0.25,0.10,0.05,0.01$ and $a=0.5$ and $\alpha=1.0)$.

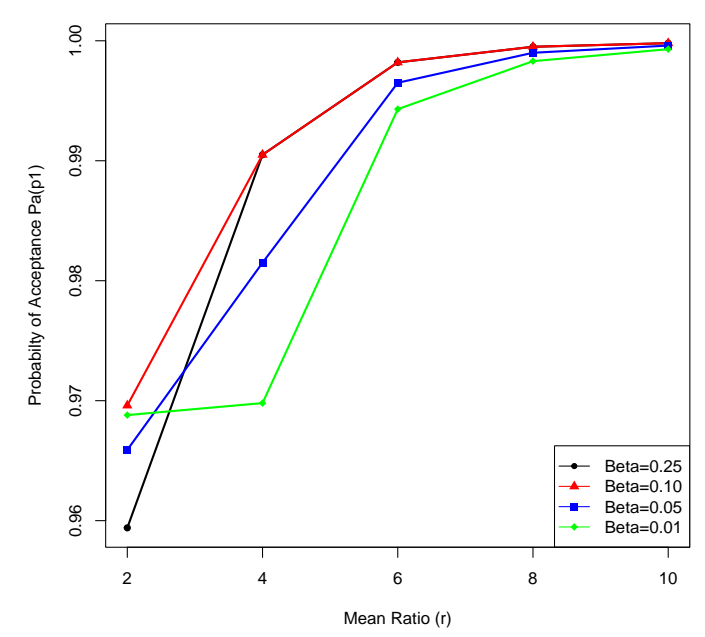

(a) $\delta=0.7, \eta=3$ and $a=0.5$

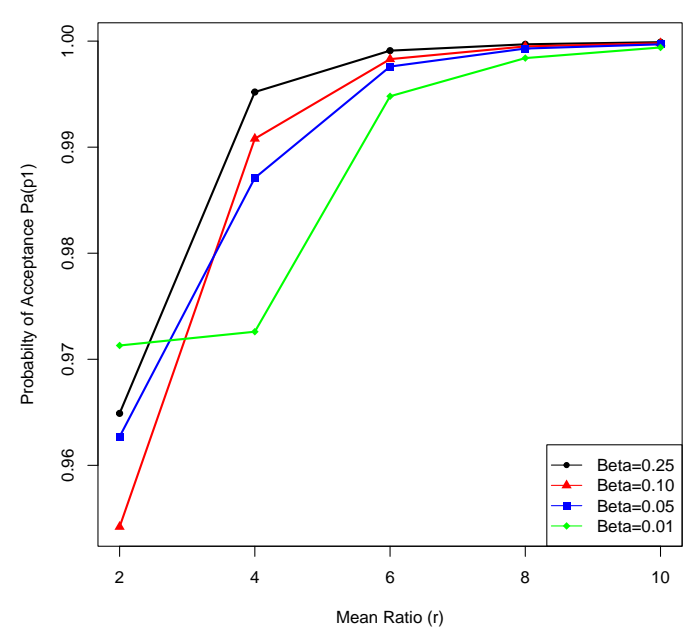

(b) $\delta=0.7, \eta=3$ and $a=1.0$

Figure 2. OC values versus mean ratio

\section{Comparative study}

This research comprises the relative comparison of the proposed plan with already existing plans in the literature. There are many types of plans under acceptance sampling procedures and similarly, there are many lifetime distributions developed in probability theory. The comparison is possible if we fix a distribution and compare the different plans of the same type based on of minimum sample size as this plan parameter makes the plan cost-efficient. Hence the comparison of the MDSRGS plan is made with both Repetitive group sampling (RGS) and Single sampling (SS) plans. Both plans follow the IPL distribution and comparison is made on the basis of ASN values as given by [3].

For baseline comparisons, the ASN at LQL which is $\left(P_{2}\right)$ is obtained for both RGS and SS plans. The calculated ASNs for SS, RGS, and MDSRGS plans with various combinations of shape parameters $\delta=0.7, \eta=3$ and $\delta=2, \eta=1.8$, are presented in Tables 7-8. Moreover, Consumers' and producers' risks, mean ratio, and experiment termination ratios are also incorporated in tables. On the other hand acceptance probabilities of the lot for the shape parameters $\delta=0.7, \eta=3$ and $\delta=2, \eta=1.8$ are calculated for the proposed and two other plans from which comparisons are made and findings are summed up in Tables 9-12. The small values of ASNs showed that the proposed MDSRGS plan is better than the RGS and SS plans as their ASNs are larger. 
Table 7. The ASNs of Proposed MDSRGS, RGS and, SS plans at $\delta=0.7, \eta=3$

\begin{tabular}{|c|c|c|c|c|c|c|c|}
\hline \multirow[b]{3}{*}{$\beta$} & \multirow[b]{3}{*}{$\frac{\mu}{\mu_{0}}$} & \multicolumn{6}{|c|}{$\delta=0.7, \eta=3$} \\
\hline & & \multicolumn{3}{|l|}{$a=0.5$} & \multicolumn{3}{|l|}{$a=1.0$} \\
\hline & & Proposed MDSRGS & RGS & SS & Proposed MDSRGS & RGS & SS \\
\hline \multirow[t]{5}{*}{0.25} & 2 & 15.2 & 17.1 & 22 & 5.1 & 5.9 & 7 \\
\hline & 4 & 9.5 & 11.5 & 12 & 3.5 & 4 & 4 \\
\hline & 6 & 9.4 & 11.5 & 12 & 3.5 & 4 & 4 \\
\hline & 8 & 9.4 & 11.5 & 12 & 3.5 & 4 & 4 \\
\hline & 10 & 9.4 & 11.5 & 12 & 3.5 & 4 & 4 \\
\hline \multirow[t]{5}{*}{0.10} & 2 & 19 & 26.3 & 34 & 8.2 & 9.4 & 14 \\
\hline & 4 & 12.3 & 13.7 & 16 & 3.5 & 4 & 5 \\
\hline & 6 & 12.2 & 13.7 & 16 & 3.5 & 4 & 5 \\
\hline & 8 & 12.2 & 13.7 & 14 & 3.5 & 4 & 4 \\
\hline & 10 & 12.2 & 13.7 & 14 & 3.5 & 4 & 4 \\
\hline \multirow[t]{5}{*}{0.05} & 2 & 26.3 & 27.6 & 44 & 8.7 & 9.4 & 16 \\
\hline & 4 & 14.2 & 15.1 & 20 & 4.4 & 4.6 & 6 \\
\hline & 6 & 14 & 15.1 & 20 & 4.3 & 4.6 & 6 \\
\hline & 8 & 14 & 15.1 & 16 & 4.3 & 4.6 & 6 \\
\hline & 10 & 14 & 15.1 & 16 & 4.3 & 4.6 & 6 \\
\hline \multirow[t]{5}{*}{0.01} & 2 & 36.6 & 35.3 & 67 & 13.2 & 11.9 & 22 \\
\hline & 4 & 19.9 & 19.9 & 35 & 5.3 & 5.3 & 10 \\
\hline & 6 & 19.5 & 19.9 & 27 & 5.2 & 5.3 & 8 \\
\hline & 8 & 19.5 & 19.9 & 27 & 5.2 & 5.3 & 8 \\
\hline & 10 & 19.4 & 19.9 & 27 & 5.2 & 5.3 & 6 \\
\hline
\end{tabular}

Table 8. The ASNs of Proposed MDSRGS, RGS and SS plans at $\delta=2, \eta=1.8$.

\begin{tabular}{|c|c|c|c|c|c|c|c|}
\hline \multirow[b]{3}{*}{$\beta$} & \multirow[b]{3}{*}{$\frac{\mu}{\mu_{0}}$} & \multicolumn{6}{|c|}{$\delta=2, \eta=1.8$} \\
\hline & & \multicolumn{3}{|l|}{$\mathrm{a}=0.5$} & \multicolumn{3}{|l|}{$\mathrm{a}=1.0$} \\
\hline & & Proposed MDSRGS & RGS & SS & Proposed MDSRGS & RGS & SS \\
\hline \multirow[t]{5}{*}{0.25} & 2 & 8.1 & 9.4 & 12 & 7.9 & 11.9 & 13 \\
\hline & 4 & 4.8 & 6 & 7 & 2.6 & 3.2 & 3 \\
\hline & 6 & 4.8 & 6 & 7 & 2.6 & 3.2 & 3 \\
\hline & 8 & 4.8 & 6 & 7 & 2.6 & 3.2 & 3 \\
\hline & 10 & 4.8 & 6 & 7 & 2.6 & 3.2 & 3 \\
\hline \multirow[t]{5}{*}{0.10} & 2 & 10.6 & 11.6 & 18 & 12.3 & 14.2 & 19 \\
\hline & 4 & 5.8 & 6.6 & 8 & 3.4 & 3.5 & 6 \\
\hline & 6 & 5.8 & 6.6 & 6 & 3.3 & 3.5 & 4 \\
\hline & 8 & 5.8 & 6.6 & 6 & 3.3 & 3.5 & 4 \\
\hline & 10 & 5.8 & 6.6 & 6 & 3.3 & 3.5 & 4 \\
\hline \multirow[t]{5}{*}{0.05} & 2 & 13.5 & 14.6 & 23 & 13.8 & 16.2 & 25 \\
\hline & 4 & 7.5 & 8 & 10 & 3.4 & 3.5 & 6 \\
\hline & 6 & 7.5 & 8 & 8 & 3.3 & 3.5 & 5 \\
\hline & 8 & 7.5 & 8 & 8 & 3.3 & 3.5 & 4 \\
\hline & 10 & 7.5 & 8 & 8 & 3.3 & 3.5 & 4 \\
\hline \multirow[t]{5}{*}{0.01} & 2 & 19.5 & 18.5 & 35 & 17.9 & 19.2 & 35 \\
\hline & 4 & 9.3 & 9.5 & 14 & 5.1 & 5.5 & 10 \\
\hline & 6 & 9.3 & 9.5 & 10 & 4.1 & 4.2 & 6 \\
\hline & 8 & 9.3 & 9.5 & 10 & 4.1 & 4.2 & 6 \\
\hline & 10 & 9.3 & 9.5 & 10 & 4.1 & 4.2 & 5 \\
\hline
\end{tabular}

\section{Practical application}

The illustration based on the practical application of the specified plan is provided in this section given that the distribution of the shape parameter is not known. Based on the production process's previous history, the suitable values for the unknown parameters are determined. As an example, two applications of the proposed plan are discussed to have an idea of its working mechanism. One is related to survival data and the other is 
about the patient's relief time. A data set containing the survival time of 72 guinea pigs is used by [14] whereas in the second example, 20 patients who are given the pain killers are assessed to know the patients' relief time, and this data set was also used by [14].

Table 9. Acceptance probabilities of proposed MDSRGS, RGS and SS plans at $\delta=0.7, \eta=3$ and $a=0.5$

\begin{tabular}{|c|c|c|c|c|c|c|c|}
\hline \multirow{5}{*}{$\beta$} & & \multicolumn{7}{|c|}{$\delta=0.7, \eta=3$} \\
\cline { 3 - 8 } & $\frac{\mu}{\mu_{0}}$ & Proposed MDSRGS & \multicolumn{2}{|c|}{ RGS } & \multicolumn{2}{c|}{ SS } \\
\hline \multirow{7}{*}{0.25} & $P_{a}\left(p_{1}\right)$ & $P_{a}\left(p_{2}\right)$ & $P_{a}\left(p_{1}\right)$ & $P_{a}\left(p_{2}\right)$ & $P_{a}\left(p_{1}\right)$ & $P_{a}\left(p_{2}\right)$ \\
& 4 & 0.9649 & 0.2370 & 0.9612 & 0.2297 & 0.9670 & 0.2383 \\
& 6 & 0.9952 & 0.2312 & 0.9948 & 0.1875 & 0.9893 & 0.2115 \\
& 8 & 0.9997 & 0.2312 & 0.9991 & 0.1875 & 0.9663 & 0.2174 \\
& 10 & 0.9999 & 0.2312 & 0.9997 & 0.1875 & 0.9815 & 0.2174 \\
0.10 & 2 & 0.9542 & 0.2312 & 0.9999 & 0.1875 & 0.9884 & 0.2174 \\
& 4 & 0.9908 & 0.0890 & 0.9575 & 0.095 & 0.9591 & 0.093 \\
& 6 & 0.9983 & 0.0890 & 0.9982 & 0.0756 & 0.9812 & 0.0963 \\
& 8 & 0.9995 & 0.0890 & 0.9995 & 0.0756 & 0.9963 & 0.0963 \\
& 10 & 0.9998 & 0.0890 & 0.9998 & 0.0756 & 0.9807 & 0.0786 \\
\hline 0.05 & 2 & 0.9627 & 0.0417 & 0.9593 & 0.0446 & 0.9635 & 0.0486 \\
& 4 & 0.9871 & 0.0481 & 0.9853 & 0.0425 & 0.9712 & 0.042 \\
& 6 & 0.9976 & 0.0481 & 0.9974 & 0.0425 & 0.9942 & 0.042 \\
& 8 & 0.9993 & 0.0481 & 0.9992 & 0.0425 & 0.9633 & 0.0473 \\
& 10 & 0.9997 & 0.0481 & 0.9997 & 0.0425 & 0.9769 & 0.0473 \\
\hline 0.01 & 2 & 0.9713 & 0.0085 & 0.9649 & 0.0093 & 0.9651 & 0.0093 \\
& 4 & 0.9726 & 0.0087 & 0.9674 & 0.0083 & 0.9887 & 0.0083 \\
& 6 & 0.9948 & 0.0087 & 0.9942 & 0.0083 & 0.9896 & 0.0092 \\
& 8 & 0.9984 & 0.0087 & 0.9983 & 0.0083 & 0.9968 & 0.0092 \\
& 10 & 0.9994 & 0.0087 & 0.9993 & 0.0083 & 0.9636 & 0.008 \\
\hline
\end{tabular}

Table 10. Acceptance probabilities of proposed MDSRGS, RGS and SS plans at $\delta=0.7, \eta=3$ and $a=1.0$.

\begin{tabular}{|c|c|c|c|c|c|c|c|}
\hline \multirow{5}{*}{$\beta$} & & \multicolumn{7}{|c|}{$\delta=0.7, \eta=3$} \\
\cline { 3 - 8 } & $\frac{\mu}{\mu_{0}}$ & $P_{a}\left(p_{1}\right)$ & $P_{a}\left(p_{2}\right)$ & $P_{a}\left(p_{1}\right)$ & $P_{a}\left(p_{2}\right)$ & $P_{a}\left(p_{1}\right)$ & $P_{a}\left(p_{2}\right)$ \\
\hline 0.25 & 2 & 0.9594 & 0.2234 & 0.9539 & 0.2155 & 0.9506 & 0.2324 \\
& 4 & 0.9905 & 0.0837 & 0.9891 & 0.0677 & 0.9822 & 0.1454 \\
& 6 & 0.9982 & 0.0837 & 0.9981 & 0.0677 & 0.9519 & 0.1362 \\
& 8 & 0.9995 & 0.0837 & 0.9995 & 0.0677 & 0.9735 & 0.1362 \\
& 10 & 0.9998 & 0.0837 & 0.9998 & 0.0677 & 0.9834 & 0.1362 \\
\hline 0.10 & 2 & 0.9696 & 0.0599 & 0.9586 & 0.0447 & 0.9782 & 0.0999 \\
& 4 & 0.9905 & 0.0837 & 0.9891 & 0.0677 & 0.9715 & 0.0654 \\
& 6 & 0.9982 & 0.0837 & 0.9981 & 0.0677 & 0.9944 & 0.0654 \\
& 8 & 0.9995 & 0.0837 & 0.9995 & 0.0677 & 0.9606 & 0.0503 \\
& 10 & 0.9998 & 0.0837 & 0.9998 & 0.0677 & 0.9752 & 0.0503 \\
\hline 0.05 & 2 & 0.9659 & 0.0451 & 0.9586 & 0.0447 & 0.9522 & 0.0334 \\
& 4 & 0.9815 & 0.0239 & 0.9781 & 0.0212 & 0.9588 & 0.0284 \\
& 6 & 0.9965 & 0.0239 & 0.9962 & 0.0212 & 0.9917 & 0.0284 \\
& 8 & 0.9990 & 0.0239 & 0.9989 & 0.0212 & 0.9974 & 0.0284 \\
& 10 & 0.9996 & 0.0239 & 0.9996 & 0.0212 & 0.9670 & 0.0185 \\
\hline 0.01 & 2 & 0.9688 & 0.0076 & 0.9617 & 0.0075 & 0.9593 & 0.0099 \\
& 4 & 0.9698 & 0.0077 & 0.9633 & 0.0073 & 0.9839 & 0.007 \\
& 6 & 0.9943 & 0.0077 & 0.9937 & 0.0073 & 0.9850 & 0.005 \\
& 8 & 0.9983 & 0.0077 & 0.9982 & 0.0073 & 0.9953 & 0.005 \\
& 10 & 0.9993 & 0.0077 & 0.9993 & 0.0073 & 0.9590 & 0.0068 \\
\hline
\end{tabular}


Table 11. Acceptance probabilities of proposed MDSRGS, RGS and SS plans at $\delta=2, \eta=1.8$ and $a=0.5$.

\begin{tabular}{|c|c|c|c|c|c|c|c|}
\hline \multirow{5}{*}{$\beta$} & & \multicolumn{6}{|c|}{$\delta=2, \eta=1.8$} \\
\cline { 3 - 8 } & & $\mu$ & Proposed MDSRGS & \multicolumn{2}{|c|}{ RGS } & \multicolumn{2}{c|}{ SS } \\
\cline { 3 - 8 } & $\mu_{0}\left(p_{1}\right)$ & $P_{a}\left(p_{2}\right)$ & $P_{a}\left(p_{1}\right)$ & $P_{a}\left(p_{2}\right)$ & $P_{a}\left(p_{1}\right)$ & $P_{a}\left(p_{2}\right)$ \\
\hline 0.25 & 2 & 0.9620 & 0.2324 & 0.9566 & 0.1947 & 0.9600 & 0.2041 \\
& 4 & 0.9984 & 0.2296 & 0.9983 & 0.1823 & 0.9516 & 0.2051 \\
& 6 & 0.9999 & 0.2296 & 0.9999 & 0.1823 & 0.9869 & 0.2051 \\
& 8 & 1.0000 & 0.2296 & 1.0000 & 0.1823 & 0.9951 & 0.2051 \\
& 10 & 1.0000 & 0.2296 & 1.0000 & 0.1823 & 0.9977 & 0.2051 \\
\hline 0.10 & 2 & 0.9629 & 0.0825 & 0.9544 & 0.081 & 0.9528 & 0.0803 \\
& 4 & 0.9974 & 0.0964 & 0.9972 & 0.0949 & 0.9929 & 0.096 \\
& 6 & 0.9998 & 0.0964 & 0.9998 & 0.0949 & 0.9783 & 0.0713 \\
& 8 & 1.0000 & 0.0964 & 1.0000 & 0.0949 & 0.9918 & 0.0713 \\
& 10 & 1.0000 & 0.0964 & 1.0000 & 0.0949 & 0.9962 & 0.0713 \\
\hline 0.05 & 2 & 0.9666 & 0.0426 & 0.9559 & 0.0344 & 0.9591 & 0.044 \\
& 4 & 0.9946 & 0.0315 & 0.9940 & 0.0282 & 0.9889 & 0.0405 \\
& 6 & 0.9996 & 0.0315 & 0.9996 & 0.0282 & 0.9740 & 0.0421 \\
& 8 & 0.9999 & 0.0315 & 0.9999 & 0.0282 & 0.9902 & 0.0421 \\
& 10 & 1.0000 & 0.0315 & 1.0000 & 0.0282 & 0.9955 & 0.0421 \\
\hline 0.01 & 2 & 0.9700 & 0.0061 & 0.9555 & 0.0051 & 0.9589 & 0.0073 \\
& 4 & 0.9909 & 0.0096 & 0.9897 & 0.0091 & 0.9785 & 0.0066 \\
& 6 & 0.9993 & 0.0096 & 0.9993 & 0.0091 & 0.9613 & 0.0086 \\
& 8 & 0.9999 & 0.0096 & 0.9999 & 0.0091 & 0.9853 & 0.0086 \\
& 10 & 1.0000 & 0.0096 & 1.0000 & 0.0091 & 0.9932 & 0.0086 \\
\hline
\end{tabular}

Table 12. Acceptance probabilities of proposed MDSRGS, RGS and SS plans at $\delta=2, \eta=1.8$ and $a=1.0$.

\begin{tabular}{|c|c|c|c|c|c|c|c|}
\hline \multirow{5}{*}{$\beta$} & & \multicolumn{6}{|c|}{$\delta=0.7, \eta=3$} \\
\cline { 3 - 8 } & & $\mu$ & \multicolumn{2}{|c|}{ Proposed MDSRGS } & \multicolumn{2}{|c|}{ RGS } & \multicolumn{2}{c|}{ SS } \\
\cline { 3 - 8 } & $P_{a}\left(p_{1}\right)$ & $P_{a}\left(p_{1}\right)$ & $P_{a}\left(p_{1}\right)$ & $P_{a}\left(p_{1}\right)$ & $P_{a}\left(p_{1}\right)$ & $P_{a}\left(p_{1}\right)$ \\
\hline 0.25 & 2 & 0.9607 & 0.2317 & 0.9683 & 0.2393 & 0.9619 & 0.2274 \\
& 4 & 0.9863 & 0.1437 & 0.9835 & 0.1082 & 0.9636 & 0.1657 \\
& 6 & 0.9985 & 0.1437 & 0.9983 & 0.1082 & 0.9955 & 0.1657 \\
& 8 & 0.9997 & 0.1437 & 0.9997 & 0.1082 & 0.9674 & 0.0667 \\
& 10 & 0.9999 & 0.1437 & 0.9999 & 0.1082 & 0.9840 & 0.0667 \\
\hline 0.10 & 2 & 0.9666 & 0.0896 & 0.9607 & 0.088 & 0.9569 & 0.0912 \\
& 4 & 0.9604 & 0.0232 & 0.9502 & 0.0202 & 0.977 & 0.0422 \\
& 6 & 0.9955 & 0.0232 & 0.995 & 0.0202 & 0.9913 & 0.0556 \\
& 8 & 0.9992 & 0.0232 & 0.9992 & 0.0202 & 0.9674 & 0.0667 \\
& 10 & 0.9998 & 0.0232 & 0.9998 & 0.0202 & 0.984 & 0.0667 \\
\hline 0.05 & 2 & 0.9596 & 0.0281 & 0.9515 & 0.0361 & 0.9566 & 0.0372 \\
& 4 & 0.9604 & 0.0232 & 0.9502 & 0.0202 & 0.977 & 0.0422 \\
& 6 & 0.9955 & 0.0232 & 0.995 & 0.0202 & 0.9858 & 0.0177 \\
& 8 & 0.9992 & 0.0232 & 0.9992 & 0.0202 & 0.9516 & 0.0172 \\
& 10 & 0.9998 & 0.0232 & 0.9998 & 0.0202 & 0.9761 & 0.0172 \\
\hline 0.01 & 2 & 0.9516 & 0.0074 & 0.957 & 0.0056 & 0.9603 & 0.0086 \\
& 4 & 0.9936 & 0.0078 & 0.9911 & 0.0061 & 0.9795 & 0.0043 \\
& 6 & 0.9911 & 0.0049 & 0.9899 & 0.0047 & 0.9793 & 0.0054 \\
& 8 & 0.9984 & 0.0049 & 0.9983 & 0.0047 & 0.9961 & 0.0054 \\
& 10 & 0.9996 & 0.0049 & 0.9996 & 0.0047 & 0.9683 & 0.0045 \\
\hline
\end{tabular}




\section{Example 1}

With the help of the survival data, the application of the acceptance sampling plan is quite interested and important. The complete methodology of the MDSRGS plan is also reported given that the shape parameter is not known. Process manufacturing history is also utilized. The data listed below comprise of survival time of 72 guinea pigs that were exposed to the infection named virulent tubercle virus.

$0.1,0.33,0.44,0.56,0.59,0.59,0.72,0.74,0.92,0.93,0.96,1,1,1.02,1.05,1.07,1.08,1.08,1.08$, $1.09,1.12,1.13,1.15,1.16,1.2,1.21,1.22,1.22,1.24,1.3,1.34,1.36,1.39,1.44,1.46,1.53,1.59$, $1.6,1.63,1.63,1.68,1.71,1.72,1.76,1.83,1.95,1.96,1.97,2.02,2.13,2.15,2.16,2.22,2.3,2.31$, $2.4,2.45,2.51,2.53,2.54,2.54,2.78,2.93,3.27,3.42,3.47,3.61,4.02,4.32,4.58,5.55$.

Table 13. Statistical measures of 72 guinea pigs data for different probability models.

\begin{tabular}{|c|c|c|c|c|c|c|}
\hline Models & MLEs & AIC & CAIC & BIC & HQIC & $K-S$ \\
\hline IPL & $\delta=0.697, \lambda=0.13, \eta=3.464$ & 193.055 & 193.398 & 199.885 & 195.774 & 0.074 \\
IL & $\lambda=12.907, \eta=0.096$ & 242.822 & 242.996 & 247.375 & 244.635 & 0.999 \\
L & $\delta=182.421, n=103.511$ & 230.535 & 230.704 & 235.089 & 232.348 & 0.0690 \\
PL & $\delta=1.709, \lambda=6.197, \eta=2.574$ & 193.075 & 193.418 & 199.905 & 195.794 & 0.078 \\
EL & $\delta=182.421, \lambda=103.5, \eta=78.322$ & 194.569 & 194.912 & 201.399 & 197.288 & 0.094 \\
GIW & $\delta=1.069, \eta=1.173, \lambda=14.374$ & 240.332 & 240.501 & 244.885 & 242.145 & 0.197 \\
\hline
\end{tabular}

It has been shown in Table 13 that the Inverse Power Lomax distribution is best fitted to the data than other candidate distributions. For the combination of shape parameters $\delta=0.7$ and $\eta=3$ the maximum likelihood estimates are also presented. The assumption that we have made is that survival meantime $\mu_{0}$ of the guinea pig is two days whereas the experiment time is one day. Moreover, the procedure termination ratio is $a=0.5$. Thus the ratio $\frac{\mu}{\mu_{0}}=4$, is the ratio for the true and specified mean, consumer's risk $(\beta)$ and producer's risks are 0.01 and 0.05 , respectively. Keeping in mind all these assumptions Table 1 has been used to demonstrate the optimal parameters for the MDSRGS plan as $n=19, c_{1}=0, c_{2}=1, m=1$.

The procedure for its implementation is given below: A life test is applied to a random sample of 19 pigs. Their survival time is then accordingly recorded (in days) as

$$
\text { 1.02, 0.92, 2.02, 2.16, 0.56, 4.02, 3.42, 1.44, 0.44, 4.02, 0.72, 3.27, 5.55, 0.56, 3.47, 1.39, }
$$

$$
0.1,0.93,4.32
$$

It is observed that 7 of the pigs do not survive for a single day. So, in this current experiment $\mathrm{d}=7$ for failed guinea pigs which shows that the failure number is more than the maximum failures such as $d>c_{2}$ i.e. $(d>6)$. Based on such findings under plan parameters, it is suggested to terminate the test and reject the claim.

\section{Example 2}

The following data set outlines the application of the proposed plan with the help of twenty patients who are given the analgesic and their relief time is recorded. The relief time is represented in minutes. To deal with the situation preceding the history of the manufacturing process is used. Besides, the complete execution procedure of the MDSRGS plan is elaborated. The relief time at various time points (in hours) is taken into account. The data set comprises the relief time of 20 patients.

$$
1.1,1.4,1.3,1.7,1.9,1.8,1.6,2.2,1.7,2.7,4.1,1.8,1.5,1.2,1.4,3.0,1.7,2.3,1.6,2.0
$$

It is observed with the help of model selection criteria that IPL distribution is best suitable to the specified data and the results of MLE for shape parameters $\delta=30, \eta=4.1$ also reflect the same (Table 14). For patient's relief time, the assumed mean time $\mu_{0}$ is 2 hours and the same for the experiment time $t_{0}$. The experiment expiry ratio is $a=1.0$ for 
this test. Table 15 shows the plan parameters of the proposed MDSRGS plan at different levels of mean ratio $\left(\frac{\mu}{\mu_{0}}\right)$ and consumer's risk $(\beta)$.

Table 14. Statistical measures of relief time of 20 patients for different probability models.

\begin{tabular}{|c|c|c|c|c|c|c|}
\hline Models & MLEs & AIC & CAIC & BIC & HQIC & $K-S$ \\
\hline IPL & $\delta=30.373, \lambda=4.77, \eta=4.067$ & 37.132 & 38.465 & 40.119 & 37.715 & 0.096 \\
IL & $\lambda=71.866, \eta=0.024$ & 69.708 & 70.414 & 71.700 & 70.097 & 0.995 \\
L & $\delta=67.937, \lambda=35.789$ & 70.263 & 70.895 & 72.254 & 70.652 & 0.872 \\
PL & $\delta=0.396, \lambda=33.79, \eta=9.367$ & 37.440 & 38.774 & 40.428 & 38.024 & 0.085 \\
EL & $\delta=76.135, \lambda=0.234, \eta=13.6$ & 37.774 & 39.107 & 40.761 & 38.357 & 0.101 \\
GIW & $\delta=3.517, \eta=3.99, \lambda=0.04$ & 37.136 & 38.469 & 40.123 & 37.719 & 0.099 \\
\hline
\end{tabular}

Table 15. The plan parameters of the proposed MDSRGS plan at $\delta=30, \eta=4.1$.

\begin{tabular}{|c|c|c|c|c|c|c|c|c|c|c|c|}
\hline$\beta$ & $\frac{\mu}{\mu_{0}}$ & \multicolumn{7}{|c|}{$\mathbf{a}=\mathbf{0 . 8}$} & \multicolumn{6}{|c|}{$\mathbf{a}=\mathbf{1 . 0}$} \\
\cline { 3 - 11 } & & $\mathbf{n}$ & $c_{1}$ & $c_{2}$ & $\mathbf{m}$ & $\mathbf{A S N}$ & $\mathbf{n}$ & $c_{1}$ & $c_{2}$ & $\mathbf{m}$ & $\mathbf{A S N}$ \\
\hline 0.25 & 2 & 5 & 0 & 1 & 2 & 6.2 & 2 & 0 & 1 & 3 & 2.9 \\
& 4 & 5 & 0 & 1 & 2 & 6.2 & 2 & 0 & 1 & 3 & 2.9 \\
& 6 & 5 & 0 & 1 & 2 & 6.2 & 2 & 0 & 1 & 3 & 2.9 \\
& 8 & 5 & 0 & 1 & 2 & 6.2 & 2 & 0 & 1 & 3 & 2.9 \\
& 10 & 5 & 0 & 1 & 2 & 6.2 & 2 & 0 & 1 & 3 & 2.9 \\
\hline 0.10 & 2 & 7 & 0 & 1 & 1 & 7.9 & 3 & 0 & 1 & 1 & 3.5 \\
& 4 & 7 & 0 & 1 & 1 & 7.9 & 3 & 0 & 1 & 1 & 3.5 \\
& 6 & 7 & 0 & 1 & 1 & 7.9 & 3 & 0 & 1 & 1 & 3.5 \\
& 8 & 7 & 0 & 1 & 1 & 7.9 & 3 & 0 & 1 & 1 & 3.5 \\
& 10 & 7 & 0 & 1 & 1 & 7.9 & 3 & 0 & 1 & 1 & 3.5 \\
\hline 0.05 & 2 & 8 & 0 & 1 & 2 & 8.8 & 4 & 0 & 1 & 1 & 4.3 \\
& 4 & 8 & 0 & 1 & 2 & 8.8 & 4 & 0 & 1 & 1 & 4.3 \\
& 6 & 8 & 0 & 1 & 2 & 8.8 & 4 & 0 & 1 & 1 & 4.3 \\
& 8 & 8 & 0 & 1 & 2 & 8.8 & 4 & 0 & 1 & 1 & 4.3 \\
& 10 & 8 & 0 & 1 & 2 & 8.8 & 4 & 0 & 1 & 1 & 4.3 \\
\hline 0.01 & 2 & 12 & 0 & 1 & 1 & 12.3 & 5 & 0 & 1 & 1 & 5.1 \\
& 4 & 12 & 0 & 1 & 1 & 12.3 & 5 & 0 & 1 & 1 & 5.1 \\
& 6 & 12 & 0 & 1 & 1 & 12.3 & 5 & 0 & 1 & 1 & 5.1 \\
& 8 & 12 & 0 & 1 & 1 & 12.3 & 5 & 0 & 1 & 1 & 5.1 \\
& 10 & 12 & 0 & 1 & 1 & 12.3 & 5 & 0 & 1 & 1 & 5.1 \\
\hline
\end{tabular}

The risk of producer and consumer are put on $\alpha=0.05$ and $\beta=0.01$ respectively. Moreover, the mean ratio is $\frac{\mu}{\mu_{0}}=2$. MDSRGS plan's design parameters are selected from Table 15 as $n=5, c_{1}=0, c_{2}=1$ and, $m=1$.

The detailed procedure of the proposed plan is as follows:

At first, 5 patients are randomly picked up from the lot, and then on selected patients, a life test is applied. Relief time of the selected patients is observed accordingly as 1.6, $2.2,1.8,1.1,2.7$

Those patients who couldn't recover within the time limit are also recorded and denoted by $d$. Three patients are not recovered before termination time $t_{0}=2$ so $d=3$. Hence it is clear that $d>c_{2}$ i.e. $(d>1)$ meaning that at the pre-defined time the total number of patients who failed to recover is more than the maximum number of patients with failed recovery. So, the highlighted conclusion is the rejection of the hypothesis immediately according to the plan parameters that terminated the test with $\alpha=5$ which is the producer's risk. 


\section{Conclusion}

The newly proposed MDSRGS plan is used when the lifetime of a product has the Inverse Power Lomax Distribution. Design parameters and ASN are found to be the two substantial factors of the MDSRGS plan. Under the theoretical distribution of the proposed plan which is Inverse Power Lomax distribution, the values of these two defined factors are estimated given that both consumer and producer risks satisfy with the lowest value of the average sample number. By employing the design parameters and OC function the sensitivity of the MDSRGS plan is also investigated. At different levels of producer's risk $(\beta)$, customer's risk $(\alpha)$, mean ratio $(r)$, and experiment termination ratio $(a)$, different values of optimal parameters i.e., $n, c_{1}, c_{2}, m$, and ASN are calculated and tabulated with the condition that the product's lifetime follows an Inverse Power Lomax distribution. Furthermore, the lot probabilities of acceptance under the proposed sampling plan are also calculated by taking different levels of producer's risk $(\beta)$, customer's risk $(\alpha)$, mean ratio $(r)$, and experiment termination ratio $(a)$.

MDSRGS plan has been compared with already existing plans known as RGS and SS plans introduced by [3] under the same distribution. In the present study, calculations of ASN at LQL i.e. $\left(p_{2}\right)$ have been performed and compared. Comparisons showed a significant result as the proposed plan has minimum values of ASN than RGS and SS plans. It is also found that the proposed plan is better in terms of minimum cost and time of inspection.

Two real-life applications of different areas (industrial and survival data) are used to provide sufficient evidence about the efficiency of the operating process of the proposed plan. Lastly, it is concluded that the rejection and acceptance of the specified lot are heavily based on the parameters and experiment ending time of the plan.

\section{Recommendation for future research}

(1) As the new sampling plan, MDSRGS is developed given that the theoretical distribution of the product's life is Inverse Power Lomax distribution. This research can be further extended using different available lifetime distributions such as Burr XII distribution, Generalized Exponential distribution, and Pareto distribution. Moreover, comparisons can also provide meaningful results to support the study.

(2) We have used the mean ratio for the estimation of the design parameters of the proposed MDSRGS plan. In light of this, the study can further be extended by using the median lifetime of a product for estimation.

(3) For inspection purposes that evaluates a one-sided capability index, the proposed plan can be made.

(4) For developing the facts about the average life of a product, the economic design of the MDSRGS plan can be introduced.

Acknowledgment. The authors are deeply thankful to the editor and reviewers for their valuable suggestions to improve the quality of the paper.

\section{References}

[1] A.I. Al-Omari, Acceptance sampling plans based on truncated lifetime tests for transmuted inverse Rayleigh distribution, Econ. Qual. Control, 31 (2), 85-91, 2016.

[2] M. Aslam, M. Azam and C.H. Jun, Multiple dependent state repetitive group sampling plan for Burr XII distribution, Qual. Eng. 28 (2), 231-237, 2016.

[3] M. Aslam, C.H. Jun, A.J. Fernández, M. Ahmad and M. Rasool, Repetitive group sampling plan based on truncated tests for Weibull models, Res. J. Appl. Sci. 7 (10), 1917-1924, 2014. 
[4] M. Aslam, D. Kundu and M. Ahmad, Time truncated acceptance sampling plans for the generalized exponential distribution, J. Appl. Stat. 37 (4), 555-566, 2010.

[5] M. Aslam, Y.L. Lio and C.H. Jun, Repetitive acceptance sampling plans for Burr type XII percentiles, Int. J. Adv. Manuf. Syst. 68 (1-4), 495-507, 2013.

[6] N. Balakrishnan, V. Leiva and J. Lopez, Acceptance sampling plans from truncated life tests based on the generalized BirnbaumSaunders distribution, Comm. Statist. Simulation Comput. 36 (3), 643-656, 2007.

[7] S. Balamurali, P. Jeyadurga and M. Usha, Designing of Bayesian multiple deferred state sampling plan based on gammaPoisson distribution, Am. J. Math. Manag. 35 (1), 77-90, 2016.

[8] S. Balamurali, P. Jeyadurga and M. Usha, Designing of multiple deferred state sampling plan for generalized inverted exponential distribution, Seq. Anal. 36 (1), 76-86, 2017.

[9] S. Balamurali, P. Jeyadurga and M. Usha, Optimal designing of multiple deferred state sampling plan for assuring percentile life under Weibull distribution, Int. J. Adv. Manuf. Syst. 93 (9-12), 3095-3109, 2017.

[10] S. Balamurali, P. Jeyadurga and M. Usha, Optimal design of repetitive group sampling plans for Weibull and gamma distributions with applications and comparison to the BirnbaumSaunders distribution, J. Appl. Stat. 45 (14), 2499-2520, 2018.

[11] H.F. Dodge, Notes on the evolution of acceptance sampling plans part I, J. Qual. Technol. 1 ( $\sup 1), 77-88,1969$.

[12] W. Gui, Double acceptance sampling plan for time truncated life tests based on Maxwell distribution, Am. J. Math. Manag. 33 (2), 98-109, 2014.

[13] W. Gui and M. Aslam, Acceptance sampling plans based on truncated life tests for the weighted exponential distribution, Comm. Statist. Simulation Comput. 46 (3), 2138-2151, 2017.

[14] A.S. Hassan and M. Abd-Allah, On the Inverse Power Lomax distribution, Ann. Data. Sci. 6, 259-278, 2019.

[15] M.A. Pawan Teh, N. Aziz and Z. Zain, Time truncated group chain sampling plans for Rayleigh distribution, Glob. J. Pure Appl. Math. 12 (4), 3693-3699, 2016.

[16] B.R. Samuel, S. Balamurali and M. Aslam, Designing of repetitive group sampling plan under truncated life test based on generalized inverted exponential distribution, J. Stat. Manag. Syst. 21(6), 955-970, 2018.

[17] R.E. Sherman, Design and evaluation of a repetitive group sampling plan, Technometrics 7 (1), 11-21, 1965.

[18] N. Singh, N. Singh and H. Kaur, Acceptance sampling plan for truncated life test having generalized Pareto distribution, Reliab. Eng. Syst. Saf. 8, 151-156, 2019.

[19] N. Singh, A. Sood and G.S. Buttar, Design of multiple deferred state repetitive group sampling plan for inverse Weibull distribution based on life test, International Journal of Scientific Research and Review 7 (3), 3734-3742, 2019.

[20] A.W. Wortham and R.C. Baker, Multiple deferred state sampling inspection, Int. J. Prod. Res. 14 (6), 719-731, 1976.

[21] C.W. Wu and Z.H. Wang, Developing a variables multiple dependent state sampling plan with simultaneous consideration of process yield and quality loss, Int. J. Prod. Res. 55 (8), 2351-2364, 2017.

[22] C.H. Yen, C.H. Chang, M. Aslam and C.H. Jun, Multiple dependent state repetitive sampling plans based on one-sided process capability indices, Comm. Statist. Theory Methods, 47 (6), 1403-1412, 2017. 